\title{
A NOTE ON THE \\ CHARACTERIZATION OF THE \\ NEOCLASSICAL PRODUCTION \\ FUNCTION
}

\author{
ANDREAS IRMEN \\ University of Luxembourg \\ and \\ CESifo, Munich
}

AlfRED MAUSSNER
University of Augsburg

We study production functions with capital and labor as arguments that exhibit positive, yet diminishing marginal products and constant returns to scale. We show that such functions satisfy the Inada conditions if (i) both inputs are essential and (ii) an unbounded quantity of either input leads to unbounded output. This allows for an alternative characterization of the neoclassical production function that altogether dispenses with the Inada conditions. Although this proposition generalizes to the case of $n>2$ factors of production, its converse does not hold: $2 n$ Inada conditions do not imply that each factor is essential.

Keywords: Neoclassical Growth Model, Capital Accumulation, Inada Conditions

\section{INTRODUCTION}

The neoclassical production function is a cornerstone of neoclassical growth theory and of modern dynamic macroeconomics in general. According to Barro and Sala-1-Martin (2004, Chap. 1), a production function taking capital and labor as arguments is called neoclassical if it exhibits three defining properties: positive and diminishing marginal products of both inputs, constant returns to scale, and the Inada conditions. ${ }^{1}$ Although the first two properties have convincing intuitive

\footnotetext{
This paper is a revised version of our CESifo Discussion Paper "Essential Inputs and Unbounded Output: An Alternative Characterization of the Neoclassical Production Function." We are grateful to the editor, two anonymous referees, Hendrik Hakenes, Amer Tabaković, and Gautam Tripathi for useful comments and suggestions. Andreas Irmen gratefully acknowledges financial support from the University of Luxembourg under the program "Agecon C-Population Aging: An Exploration of its Effect on Economic Performance and Culture." Address correspondence to: Alfred Maußner, Chair of Empirical Macroeconomics, University of Augsburg, Universitätsstrasse 16, D-86316 Augsburg, Germany; e-mail: alfred.maussner@wiwi.uni-augsburg.de.
} 
appeal, the main justification given for the Inada conditions is simply analytical convenience. $^{2}$

The main purpose of this note is to show that an intuitive justification can be given for the Inada conditions. More precisely, our main result applies to production functions that possess the first two properties. For such functions, we show that the Inada conditions hold if both capital and labor are essential and if an unbounded quantity of either input leads to an unbounded output. Using this finding, we come up with an alternative, yet equivalent, definition of the neoclassical production function that altogether dispenses with the Inada conditions. ${ }^{3}$

We develop our main result in three steps. First, we show in Section 2 that the Inada condition at infinity for one input is implied by the essentiality of the other input. Second, we establish sufficient conditions for the Inada conditions at zero in Section 3. They include the condition of essential inputs in conjunction with the requirement that output becomes unbounded as either input goes to infinity. Finally, Section 4 has our main result. The (four) Inada conditions are shown to be equivalent to a set of four conditions: capital and labor are essential and an unbounded input of either of these inputs leads to an unbounded output. We use this finding for an alternative characterization of the neoclassical production function.

In Section 5, we ask whether the previous results extend to the case of $n>2$ factors of production. Here, we first generalize the conditions that imply the Inada conditions at infinity and at zero. Second, we prove that all $2 n$ Inada conditions hold if all factors of production are essential and an unbounded input of each of them leads to an unbounded output. However, we also find that the converse of this statement is not true. Therefore, a characterization of the neoclassical production function without reference to the Inada conditions seems more natural than the one given by Barro and Sala-1́-Martin (2004).

\section{ESSENTIAL INPUTS AND THE INADA CONDITIONS AT INFINITY}

Consider an aggregate production function $F(K, L)$, where $K>0$ and $L>0$ denote the inputs of capital and labor. This function has the following properties.

DEFINITION 1 (Aggregate Production Function)

The aggregate production function, $F: \mathbf{R}_{++}^{2} \rightarrow \mathbf{R}_{++}$,

1. is twice differentiable with positive, yet diminishing marginal products, i.e.,

$$
F_{K}(K, L)>0>F_{K K}(K, L) \quad \text { and } \quad F_{L}(K, L)>0>F_{L L}(K, L) ;
$$

2. exhibits constant returns to scale in $K$ and $L$.

For functions that comply with Definition 1, we now establish that essential inputs are sufficient for the Inada conditions at infinity. To introduce the notion of an essential input, let $F(K, 0) \equiv \lim _{L \rightarrow 0} F(K, L)$ and 
$F(0, L) \equiv \lim _{K \rightarrow 0} F(K, L)$. Then labor is an essential input, or essential for short, if $F(K, 0)=0$, and capital is essential if $F(0, L)=0$.

PROPOSITION 1 (Essential Inputs and Inada Conditions at Infinity)

For an aggregate production function of Definition 1 the following hold:

1. If labor is essential then $\lim _{K \rightarrow \infty} F_{K}(K, L)=0$.

2. If capital is essential then $\lim _{L \rightarrow \infty} F_{L}(K, L)=0$.

Proof of Proposition 1. The proof of Proposition 1 involves an intuitive argument for each claim. For brevity we focus on Claim 1. Mutatis mutandis, the argument is analogous for Claim 2 [see Irmen and Maußner (2014)].

Because labor is essential, the average product of capital must vanish as labor converges to zero. ${ }^{4}$ To see this, let $k \equiv K / L$ and $F(k, 1) \equiv f(k)$. Because $(K, L) \in \mathbf{R}_{++}^{2}$, we have $k \in \mathbf{R}_{++}$. Thus, it holds that

$$
\lim _{L \rightarrow 0} F(K, L)=K \lim _{L \rightarrow 0} \frac{F(K, L)}{K}=K \lim _{L \rightarrow 0} \frac{f(k)}{k} .
$$

From the definition of $k$ it follows that $\lim _{L \rightarrow 0} k=\lim _{k \rightarrow \infty} k=\infty$. Hence, if labor is essential, then

$$
0=\lim _{L \rightarrow 0} \frac{f(k)}{k}=\lim _{k \rightarrow \infty} \frac{f(k)}{k} .
$$

By assumption, the marginal product of labor is strictly positive, so that $F_{L}(K, L)=f(k)-k f^{\prime}(k)>0$. Accordingly, the average product of capital must be strictly greater than its marginal product; i. e., $f(k) / k>f^{\prime}(k)$. Then, with (1), we obtain

$$
0=\lim _{k \rightarrow \infty} \frac{f(k)}{k} \geq \lim _{k \rightarrow \infty} f^{\prime}(k)=\lim _{K \rightarrow \infty} F_{K}(K, L),
$$

where the last equality follows because $f^{\prime}(k)=F_{K}(K, L)$ for all $K$ and $L$. In other words, as $f(k) / k$ tends to zero as $L \rightarrow 0, f^{\prime}(k)=F_{K}(K, L)$ must also tend to zero. Accordingly, the Inada condition at infinity for capital is satisfied if labor is essential.

Hence, essentiality of one input implies the Inada condition at infinity for the other input. To illustrate Proposition 1, consider the common CES production function

$$
F(K, L)=\left\{\begin{array}{cc}
A\left(\alpha K^{\frac{\sigma-1}{\sigma}}+(1-\alpha) L^{\frac{\sigma-1}{\sigma}}\right)^{\frac{\sigma}{\sigma-1}} & \sigma \neq 1, \\
A K^{\alpha} L^{1-\alpha} & \sigma=1,
\end{array}\right.
$$

where $A>0,0<\alpha<1$, and $\sigma>0$ is the elasticity of substitution. If $\sigma \leq 1$ then labor is essential and hence the marginal product of capital vanishes as $K \rightarrow \infty$. The "symmetry" of the CES also implies that capital is essential if 
$\sigma \leq 1$. Hence, the marginal product of labor also vanishes as $L \rightarrow \infty$. If $\sigma>1$ then neither labor nor capital is essential, and the contrapositive of Proposition 1 holds true, as $\lim _{K \rightarrow \infty} F_{K}(K, L)=A \alpha^{\sigma /(\sigma-1)}>0$ and $\lim _{L \rightarrow \infty} F_{L}(K, L)=$ $A(1-\alpha)^{\sigma /(\sigma-1)}>0 .^{5}$

\section{UNBOUNDED OUTPUT, ESSENTIAL INPUTS, AND THE INADA CONDITIONS AT ZERO}

In addition to essential inputs, the Inada conditions at zero require that output approaches infinity as either input goes to infinity. We capture this property using the following notation: ${ }^{6} F(K, \infty) \equiv \lim _{L \rightarrow \infty} F(K, L)=\infty$ and $F(\infty, L) \equiv$ $\lim _{K \rightarrow \infty} F(K, L)=\infty$.

PROPOSITION 2 (Unbounded Output, Essential Inputs, and Inada Conditions at Zero)

For an aggregate production function of Definition 1 the following hold:

1. If $F(K, \infty)=\infty$ and capital is essential then $\lim _{K \rightarrow 0} F_{K}(K, L)=\infty$.

2. If $F(\infty, L)=\infty$ and labor is essential then $\lim _{L \rightarrow 0} F_{L}(K, L)=\infty$.

Proof of Proposition 2. As before, the proof of Proposition 2 involves an intuitive argument for each claim. For brevity, we focus on Claim 1. Mutatis mutandis, the argument is analogous for Claim 2 [see Irmen and Maußner (2014)].

If output becomes unbounded as labor goes to infinity, then the average product of capital becomes unbounded, too; i. e., for $L \rightarrow \infty$ we have $F(K, L) / K \rightarrow \infty$. Under constant returns to scale, the latter implies that $f(k) / k \rightarrow \infty$ as $k \rightarrow 0$. To see this formally, observe that for any $K \in \mathbf{R}_{++}$it holds that

$$
\lim _{L \rightarrow \infty} F(K, L)=K \lim _{L \rightarrow \infty} \frac{F(K, L)}{K}=K \lim _{L \rightarrow \infty} \frac{f(k)}{k} .
$$

Recall that $\lim _{L \rightarrow \infty} k=\lim _{k \rightarrow 0} k=0$. Hence, if output approaches infinity as labor goes to infinity, then

$$
\infty=\lim _{L \rightarrow \infty} \frac{f(k)}{k}=\lim _{k \rightarrow 0} \frac{f(k)}{k} .
$$

Because capital is essential, $\lim _{k \rightarrow 0} f(k)=0$, we may apply L'Hôpital's rule to evaluate the rightmost limit in (3), yielding

$$
\lim _{k \rightarrow 0} \frac{f(k)}{k}=\lim _{k \rightarrow 0} f^{\prime}(k)=\lim _{K \rightarrow 0} F_{K}(K, L)=\infty .
$$

Hence, the Inada condition at zero for one input obtains if this input is essential and output becomes unbounded as the other input tends to infinity.

One readily verifies that the CES production function of (2) is incompatible with Proposition 2 unless $\sigma=1$. Indeed, if $\sigma<1$, then $F(K, \infty)=A K \alpha^{\sigma /(\sigma-1)}<\infty$ 
and $F(\infty, L)=A L(1-\alpha)^{\sigma /(\sigma-1)}<\infty$. At the same time, both inputs are essential and both Inada conditions at zero are violated; i. e., $\lim _{K \rightarrow 0} F_{K}(K, L)=$ $A \alpha^{\sigma /(\sigma-1)}<\infty$ and $\lim _{L \rightarrow 0} F_{L}(K, L)=A(1-\alpha)^{\sigma /(\sigma-1)}<\infty$. If $\sigma>1$ then both $F(K, \infty)=F(\infty, L)=\infty$, yet neither capital nor labor is essential, as $F(K, 0)=A K \alpha^{\sigma /(\sigma-1)}>0$ and $F(0, L)=A L(1-\alpha)^{\sigma /(\sigma-1)}>0$. However, both Inada conditions at zero hold.

\section{AN ALTERNATIVE CHARACTERIZATION OF THE NEOCLASSICAL PRODUCTION FUNCTION}

What are the implications of Proposition 1 and 2 for the notion of a neoclassical production function? The formal definition of this concept is the following [see Barro and Sala-1-Martin (2004, pp. 27-28)].

\section{DEFINITION 2 (Neoclassical Production Function)}

An aggregate production function of Definition 1 is called a neoclassical production function if it also satisfies the Inada conditions

$$
\begin{aligned}
& \lim _{K \rightarrow 0} F_{K}(K, L)=\lim _{L \rightarrow 0} F_{L}(K, L)=\infty, \\
& \lim _{K \rightarrow \infty} F_{K}(K, L)=\lim _{L \rightarrow \infty} F_{L}(K, L)=0 .
\end{aligned}
$$

To establish an alternative and equivalent definition to Definition 2, we first use Proposition 1 and 2 to state and prove the following result.

PROPOSITION 3 (Essential Inputs, Unbounded Output, and Inada Conditions)

An aggregate production function of Definition 1 satisfies the set of Inada conditions (4) if and only if it satisfies

$$
\begin{gathered}
F(0, L)=F(K, 0)=0, \\
F(K, \infty)=F(\infty, L)=\infty .
\end{gathered}
$$

Proof of Proposition 3. The proof and the intuition behind Proposition 3 are straightforward. In fact the "if" part follows directly from Proposition 1 and 2. From Proposition 1 we know that $F(0, L)=F(K, 0)=0$ implies that $\lim _{K \rightarrow \infty} F_{K}(K, L)=\lim _{L \rightarrow \infty} F_{L}(K, L)=0$. Proposition 2 states that the same essentiality conditions in conjunction with unbounded output, i.e., $F(K, \infty)=$ $F(\infty, L)=\infty$, imply that $\lim _{K \rightarrow 0} F_{K}(K, L)=\lim _{L \rightarrow 0} F_{L}(K, L)=\infty$. Moreover, the converse implications are established in Barro and Sala-í-Martin (2004, pp. 77-78). Accordingly, Proposition 3 holds.

Hence, an aggregate production function of Definition 1 will satisfy the Inada conditions if and only if both inputs are essential and output becomes unbounded if one input becomes unbounded. Proposition 3 leads to the main result of this 
note. We state our alternative definition of the neoclassical production function as Corollary 1.

COROLLARY 1 (Neoclassical Production Function without Inada Conditions)

Consider an aggregate production function of Definition 1. This function is a neoclassical production function in the sense of Definition 2 if it satisfies the conditions (5).

Hence, the alternative definition of the neoclassical production function that we propose replaces the four Inada conditions with four new conditions: each input is essential and output becomes unbounded if either input tends to infinity.

\section{THE NEOCLASSICAL PRODUCTION FUNCTION WITH $n>2$ FACTORS OF PRODUCTION}

Do the results of the preceding analysis generalize to the case of $n>2$ factors of production? Toward this purpose we introduce the following notation. Let $\mathbf{X}=$ $\left(X_{1}, X_{2}, \ldots, X_{n}\right) \in \mathbf{R}_{++}^{n}$ be a vector of $n>2$ factors of production. Moreover, denote first- and second-order partial derivatives by $\partial F(\mathbf{X}) / \partial X_{i} \equiv F_{i}(\mathbf{X})$ and $\partial^{2} F(\mathbf{X}) / \partial X_{i}^{2} \equiv F_{i i}(\mathbf{X})$, respectively. Then $F(\mathbf{X})$ is the aggregate production function and has the following properties.

DEFINITION 3 (Aggregate Production Function with $n>2$ Factors of Production)

The aggregate production function, $F: \mathbf{R}_{++}^{n} \rightarrow \mathbf{R}_{++}$,

1. is twice differentiable with positive, yet diminishing marginal products, i.e.,

$$
F_{i}(\mathbf{X})>0>F_{i i}(\mathbf{X}) \quad \text { for all } i=1,2, \ldots, n ;
$$

2. exhibits constant returns to scale in $\mathbf{X}$.

The following proposition establishes the implications of essentiality and unbounded output for the Inada conditions. To accomplish this let $\mathbf{X}_{-i}=$ $\left(X_{1}, X_{2}, \ldots, X_{i-1}, X_{i+1}, \ldots, X_{n}\right)$. Then, analogously to our previous notation, we have $F\left(\mathbf{X}_{-i}, 0\right) \equiv \lim _{X_{i} \rightarrow 0} F(\mathbf{X})$, so that $X_{i}$ is essential if $F\left(\mathbf{X}_{-i}, 0\right)=0$. In the same vein, let $F\left(\mathbf{X}_{-i}, \infty\right) \equiv \lim _{X_{i} \rightarrow \infty} F(\mathbf{X})$. Then $F\left(\mathbf{X}_{-i}, \infty\right)=\infty$ means that output approaches infinity as $X_{i}$ goes to infinity.

PROPOSITION 4 (Essential Inputs, Unbounded Output, and Inada Conditions with $n>2$ Factors of Production)

Consider an aggregate production function of Definition 3.

1. If $X_{m}$ is essential then $\lim _{X_{i} \rightarrow \infty} F_{i}(\mathbf{X})=0$ for all $X_{i} \in \mathbf{X}_{-m}$.

2. If $F\left(\mathbf{X}_{-m}, \infty\right)=\infty$ then for each essential $X_{i} \in \mathbf{X}_{-m}$, it holds that $\lim _{X_{i} \rightarrow 0} F_{i}(\mathbf{X})=$ $\infty$. 
Proof of Proposition 4. Without loss of generality, suppose that $m=1$. Let $x_{j} \equiv X_{j} / X_{1}$ for $j=2, \ldots, n$ and $F\left(1, x_{2}, \ldots, x_{n}\right) \equiv f\left(\mathbf{x}_{-1}\right)=f\left(x_{2}, \ldots, x_{n}\right)$.

1. Proof of Claim 1

$X_{1}$ is essential. Because $\mathbf{X} \in \mathbf{R}_{++}^{n}$, we have $x_{j} \in \mathbf{R}_{++}$, and $\lim _{X_{1} \rightarrow 0} x_{j}=$ $\lim _{x_{j} \rightarrow \infty} x_{j}=\infty$. Thus, it holds that

$$
0=\lim _{X_{1} \rightarrow 0} F(\mathbf{X})=\lim _{X_{1} \rightarrow 0} X_{1} f\left(\mathbf{x}_{-1}\right)=X_{j} \lim _{x_{j} \rightarrow \infty} \frac{f\left(\mathbf{x}_{-1}\right)}{x_{j}}=\lim _{x_{j} \rightarrow \infty} \frac{f\left(\mathbf{x}_{-1}\right)}{x_{j}} .
$$

By assumption, the marginal product of $X_{1}$ is strictly positive, so that

$$
F_{1}(\mathbf{X})=f\left(\mathbf{x}_{-1}\right)-\sum_{l=2}^{n} f_{l}\left(\mathbf{x}_{-1}\right) x_{l}>0 .
$$

This implies that

$$
\frac{f\left(\mathbf{x}_{-1}\right)}{x_{j}}>f_{j}\left(\mathbf{x}_{-1}\right)+\sum_{l=2, l \neq j}^{n} f_{l}\left(\mathbf{x}_{-1}\right) \frac{X_{l}}{X_{j}} .
$$

Then, with (6), we obtain

$$
0=\lim _{x_{j} \rightarrow \infty} \frac{f\left(\mathbf{x}_{-1}\right)}{x_{j}} \geq \lim _{x_{j} \rightarrow \infty}\left(f_{j}\left(\mathbf{x}_{-1}\right)+\sum_{l=2, l \neq j}^{n} f_{l}\left(\mathbf{x}_{-1}\right) \frac{X_{l}}{X_{j}}\right) .
$$

Hence,

$$
\lim _{x_{j} \rightarrow \infty} f_{j}\left(\mathbf{x}_{-1}\right)=\lim _{X_{j} \rightarrow \infty} F_{j}(\mathbf{X})=0 \quad \text { for all } j=2,3, \ldots, n
$$

\section{Claim 2}

Because output becomes unbounded as $X_{1}$ goes to infinity, we have for all $j=$ $2,3, \ldots, n$

$$
\infty=\lim _{X_{1} \rightarrow \infty} F(\mathbf{X})=\lim _{X_{1} \rightarrow \infty} X_{1} f\left(\mathbf{x}_{-1}\right)=X_{j} \lim _{x_{j} \rightarrow 0} \frac{f\left(\mathbf{x}_{-1}\right)}{x_{j}}=\lim _{x_{j} \rightarrow 0} \frac{f\left(\mathbf{x}_{-1}\right)}{x_{j}} .
$$

Because $X_{j}$ is essential, $\lim _{x_{j} \rightarrow 0} f\left(\mathbf{x}_{-1}\right)=0$, and L'Hôpital's rule implies

$$
\infty=\lim _{x_{j} \rightarrow 0} \frac{f\left(\mathbf{x}_{-1}\right)}{x_{j}}=\lim _{x_{j} \rightarrow 0} f_{j}\left(\mathbf{x}_{-1}\right)=\lim _{X_{j} \rightarrow 0} F_{j}(\mathbf{X}),
$$

yielding the desired result.

Claim 1 generalizes Proposition 1 to the case of $n>2$ factors of production. It states that the essentiality of one factor implies the Inada condition at infinity for all other factors. Claim 2 generalizes Proposition 2 to the case of $n>2$ factors of production. In plain words, it states the following: if output becomes unbounded as the input of one factor of production tends to infinity, then the Inada condition at zero holds for all other factors that are essential. 
Observe that the converse of Claim 1 of Proposition 4 does not hold; i. e.,

$$
\lim _{X_{i} \rightarrow \infty} F_{i}(\mathbf{X})=0 \text { for all } i=1,2, \ldots, n \text { and } i \neq m \quad \nRightarrow \quad F\left(\mathbf{X}_{-m}, 0\right)=0 .
$$

To see this, consider

$$
F(\mathbf{X})=X_{1}^{\alpha} X_{2}^{1-\alpha}+X_{1}^{\alpha} X_{2}^{\beta} X_{3}^{1-\alpha-\beta}, \quad 0<\alpha<1,0<\beta<1, \alpha+\beta<1 .
$$

This function is consistent with Definition 3. Moreover, $\lim _{X_{1} \rightarrow \infty} F_{1}(\mathbf{X})=$ $\lim _{X_{2} \rightarrow \infty} F_{2}(\mathbf{X})=0$. However, $X_{3}$ is not essential, as $F\left(X_{1}, X_{2}, 0\right)=$ $X_{1}^{\alpha} X_{2}^{1-\alpha}>0$.

Proposition 4 lends itself to the following corollary.

COROLLARY 2 (Essential Inputs, Unbounded Output, and Inada Conditions with $n>2$ Factors of Production)

Consider an aggregate production function of Definition 3.

If $F\left(\mathbf{X}_{-i}, 0\right)=0$ and $F\left(\mathbf{X}_{-i}, \infty\right)=\infty$ for all $i=1,2, \ldots, n$, then $F(\mathbf{X})$ satisfies

$$
\lim _{X_{i} \rightarrow 0} F_{i}(\mathbf{X})=\infty \quad \text { and } \quad \lim _{X_{i} \rightarrow \infty} F_{i}(\mathbf{X})=0 \quad \text { for all } i=1,2, \ldots, n .
$$

In other words, an aggregate production function of Definition 3 satisfies all $2 n$ Inada conditions if all factors of production (i) are essential and (ii) deliver an unbounded output as their respective input quantities approach infinity. Observe that the converse of Corollary 2 does not hold, as $F(\mathbf{X})$ of (8) exemplifies. This function satisfies all six Inada conditions, yet $X_{3}$ is not essential. Hence, the finding of Barro and Sala-1-Martin (2004, pp. 77-78)], according to which the Inada conditions at infinity imply essential inputs is only valid for $n=2$ factors of production. We therefore propose the following generalized definition of the neoclassical production function without reference to the Inada conditions.

DEFINITION 4 (Neoclassical Production Function without Inada Conditions for $n>2$ Factors of Production)

Consider an aggregate production function of Definition 3. Such function is called a neoclassical production function if it satisfies

$$
F\left(\mathbf{X}_{-i}, 0\right)=0 \quad \text { and } \quad F\left(\mathbf{X}_{-i}, \infty\right)=\infty \quad \text { for all } i=1,2, \ldots, n .
$$

\section{NOTES}

1. Some authors, such as Acemoglu (2009, Chap. 2) and Romer (2012, Chap. 1), include the essentiality of capital in this set of properties. However, the three defining properties mentioned in the preceding can be shown to imply this feature [see Barro and Sala-í-Martin (2004, Chap. 1)].

2. The Inada conditions prescribe that the limit of the marginal products of capital and labor is infinity (zero) as the respective input approaches zero (infinity). In many circumstances, these conditions are sufficient for the existence of interior equilibria. However, they may also give rise to counterintuitive features [see, e. g., Hakenes and Irmen (2008)]. Inada himself does not provide an 
economic justification for his conditions either but simply refers to them as "derivative conditions" [Inada (1963, p. 121)].

3. The literature related to this note includes important contributions on the axiomatic foundations of aggregate production functions [see, e.g., Shephard (1970) and Färe (1980)]. However, unlike the present note, the main focus of these works is on an appropriate formal statement of the law of diminishing returns. More recently, Färe and Primont (2002) scrutinized implications of the Inada conditions and pointed to a possible incompatibility with such a statement. Contrary to this, our focus is on the set of conditions that imply the Inada conditions.

4. Burmeister and Dobell (1970, p. 25) use this property to prove the existence of a strictly positive steady-state capital intensity in the model of Solow (1956). However, these authors do not address the implications of this property for the Inada conditions at infinity.

5. To further illustrate the claims of Proposition 1, consider two "asymmetric" and less common production functions that are both consistent with Definition 1 [see de la Croix and Michel (2002, p. 122) and Litina and Palivos (2008, p. 298), respectively]. First, let $F(K, L)=b L+a L K /(L+K)$, $a, b>0$. Here, labor is essential and capital is not. Accordingly, we have $\lim _{K \rightarrow \infty} F_{K}(K, L)=$ $\lim _{K \rightarrow \infty} a(L /(L+K))^{2}=0$, whereas $\lim _{L \rightarrow \infty} F_{L}(K, L)=\lim _{L \rightarrow \infty} b+a[K /(K+L)]^{2}=b>$ 0 . Second, let $F(K, L)=A K+\alpha L-\beta L \exp (-\beta K / L), A>0, \alpha \geq \beta>0$. Here, capital is essential if $\alpha=\beta$, in which case $\lim _{L \rightarrow \infty} F_{L}(K, L)=\lim _{L \rightarrow \infty} \alpha[1-(1+\alpha K / L) \exp (-\alpha K / L)]=0$. Interestingly, for $\alpha>\beta$, capital is no longer essential and the conclusion of Claim 2 is no longer valid, as $\lim _{L \rightarrow \infty} F_{L}(K, L)=\alpha-\beta>0$.

6. In the parlance of Färe and Primont (2002), capital is said to be not limitational if $F(K, \infty)=\infty$. This wording intuitively suggests that even though the amount of capital is finite, it cannot impose a limit on the amount of output as labor becomes unbounded. Similarly, labor is said to be not limitational if $F(\infty, L)=\infty$.

\section{REFERENCES}

Acemoglu, D. (2009) Introduction to Modern Economic Growth. Princeton, NJ: Princeton University Press.

Barro, R.J. and X. Sala-1́-Martin (2004) Economic Growth, 2nd ed. Cambridge, MA: MIT Press.

Burmeister, E. and R.A. Dobell (1970) Mathematical Theories of Economic Growth. London: CollierMacmillian.

de la Croix, D. and P. Michel (2002) A Theory of Economic Growth-Dynamics and Policy in Overlapping Generations. Cambridge, UK: Cambridge University Press.

Färe, R. (1980) Laws of Diminishing Returns. Berlin: Springer.

Färe, R. and D. Primont (2002) Inada conditions and the law of diminishing returns. International Journal of Business Economics 1, 1-8.

Hakenes, H. and A. Irmen (2008) Neoclassical growth and the "trivial" steady state. Journal of Macroeconomics 30(3), 1097-1103.

Inada, K.-I. (1963) On two-sector models of economic growth: Comments and a generalization. Review of Economic Studies 30, 119-127.

Irmen, A. and A. Maußner (2014) Essential Inputs and Unbounded Output: an Alternative Characterization of the Neoclassical Production Function. CESifo working paper 5126, CESifo Group Munich.

Litina, A. and T. Palivos (2008) Do Inada conditions imply that production function must be asymptotically Cobb-Douglas? A comment. Economics Letters 99, 498-499.

Romer, D. (2012) Advanced Macroeconomics, 4th ed. New York: McGraw Hill.

Shephard, R.W. (1970) Proof of the law of diminishing returns. Zeitschrift für Nationalökonomie 30, 7-34.

Solow, R.M. (1956) A contribution to the theory of economic growth. Quarterly Journal of Economics 70(1), 65-94. 